\title{
Transmigrated canines: review and update
}

\author{
Alba $\mathrm{M}^{\mathrm{a}}$ Maceiras Pérez ${ }^{1}, \mathrm{M}^{\mathrm{a}}$ Isabel Leco Berrocal ${ }^{2}$, Cristina Barona Dorado ${ }^{3}$, Fernando Fernández Cáliz ${ }^{4}$, \\ José $M^{a}$ Martínez González ${ }^{5}$ \\ ${ }^{1}$ Student of the Master in Oral and Dental Implant Surgery at the University Hospital of Madrid. \\ ${ }^{2}$ Professor of Dentistry at the European University of Madrid. Professor of the Master's degree in Oral and Dental Implant Sur- \\ gery at the University Hospital of Madrid. \\ ${ }^{3}$ Associate Professor of Surgery at the Complutense University of Madrid. Assistant Director of the Master's degree in Oral and \\ Dental Implant Surgery at the University Hospital of Madrid. \\ ${ }^{4}$ Associate Professor of Surgery at the Complutense University of Madrid. Professor of the Master's degree in Oral and Dental \\ Implant Surgery at the University Hospital of Madrid. \\ ${ }^{5}$ Full Professor of Maxillofacial Surgery at the Complutense University of Madrid. Head of the Department of Oral and Dental \\ Implant Surgery at the University Hospital of Madrid.
}

Correspondence:

C/ Golfo de Salónica $n^{\circ} 15$

28033 Madrid, Spain

E-mail:alba_maceiras@hotmail.com

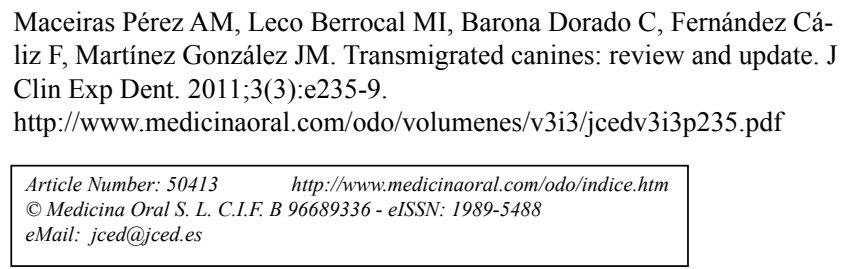

\begin{abstract}
Transmigrated canines are impacted canines that have crossed the midline.

Objective: To evaluate the epidemiological and clinical aspects that have to do with transmigrated canines.

Study design: Bibliographic review of 209 transmigrated canines obtained from a review of articles from 1971 to the present.

Results: Transmigrated canines are most frequent among females and are usually diagnosed within the first two decades of life. They are usually located in the mandible, with transmigration being most frequent in the left mandibular canine. In a large number of cases, the deciduous canine remains in the arcade. Transmigrated canines are most often found impacted, the primary position being mesially inclined, having crossed the midline. There is no pathology that is clearly associated with these canines, although radiolucence compatible with a cyst was found in a great percentage.

Conclusions: Given that it is not possible to predict when dental transmigration is going to occur, we must monitor those mandibular canines which present a delay in their eruption, in order to try to carry out an interceptive treatment and avoid possible pathologies.
\end{abstract}

Key words: transmigrated canine, dental migration, impacted canine. 


\section{Introduction}

Transmigration is described as a phenomenon in which more than half of the length of an unerupted impacted tooth has passed the midline (1-4). In the case of the canines, it is believed to start at a young age, when the patient is between 6 and 8 years old, before root formation of the canine has finished. In the majority of cases, it takes place in the mandible and usually occurs unilaterally (5). It is thought that some of the reasons why it is less common in the maxilla versus the mandible have to do with the minimum distance between the floor of the nasal cavity and the apex of the canine, or the presence of the mid-palatal suture (6).

According to different authors $(1,4)$, the factors described as contributing to the onset of this anomaly are the premature loss of deciduous teeth (occupying the space by adjacent teeth), the bone-tooth discrepancy, agenesis of lower lateral incisors, endocrinopathies and hereditary factors, trauma and odontomas, tumors, mandibular fractures, any small obstacle that may divert the canine (such as a root fragment), and some authors believe that this phenomenon is more prevalent in patients with midline deviation (2).

Clinically, transmigration usually occurs without symptoms, without any type of alteration. Upon clinical examination, one can observe the permanence of the deciduous tooth beyond its period of exfoliation, or the absence of the permanent canine in the arcade. In some cases, however, there were symptoms, as there was an associated follicular cyst or a chronic infection with fistulation (7).

Although the transmigrated canines usually do not erupt, there have been cases described in which this does occur (1).

As for treatment of these canines, ideally an interceptive treatment would be carried out, although it is almost impossible to be able to predict the onset of this anomaly $(1,8)$. To do so, it would be necessary to detect those patients between the ages of 8 and 9 years old who present the characteristics most associated with transmigration, and a clinical / X-ray exam would have to be performed in order to be able to take action quickly. Approached in this manner, there are more treatment options available, the most adequate being rescue surgery (surgical repositioning), autologous transplant or surgical-orthodontic treatment (1). This is provided that the crown of the migrated tooth is not located beyond the contralateral incisors, or the apex is not located beyond the apex of the adjacent lateral incisor, in which case, it would be mechanically impossible to reposition it in its place in the arcade $(5,9)$. In such cases, the preferred treatment would be surgical extraction. The watchful waiting approach (observation, periodic clinical checkup and Xray exam) would be a last-resort treatment option. This watchful waiting approach may be necessary if the cani- ne is in a very ectopic and asymptomatic situation, or if the patient is an overall poor state (10).

The aims of this research were to evaluate the epidemiological aspects of transmigrated canines, the frequency of appearance and the clinical manifestations, in order to obtain more predictable results.

\section{Material and Methods}

We performed a bibliographic review of the results obtained from a search in PubMed using the following keywords: transmigrant canine, tooth migration, impacted canine. From this search, we obtained references from 1971 to 2007, selecting all of the articles that fulfilled the inclusion criteria of at least four out of six of the variables mentioned below.

In accordance with this, the variables studied were as follows:

- Age: Record the age of the patient when the transmigrated canines were diagnosed.

- Sex: Record the sex of the patient who presents the transmigrated canine, and evaluate whether there is a relationship between that of the patient's sex and transmigration.

- Type of canine involved: Evaluate if there is a greater prevalence of transmigration depending on the type of canine.

- Presence or no presence of the deciduous canine: We analyzed whether there is a correlation between transmigration and exfoliation or permanence of the deciduous canine in the mouth.

- Location: Determine what is the most common position in which the transmigrated canine is found

- Associated pathology: Determine if there is a prevalence of any pathology that may be associated with this phenomenon, such as supernumerary teeth, cysts, odontomas, etc.

With all of the data obtained, we created a table in which we recorded all of the variables studied. We then performed a descriptive statistical analysis in order to evaluate the frequency and prevalence of the transmigrated canines, their location, the type of canine most commonly involved, and the presence of associated pathology, as well as the distribution by sex and the mean, range and standard deviation for the age of the patient at the time of diagnosis.

\section{Results}

A total of 209 transmigrated canines were recorded in 187 patients. The patients recorded presented from a single transmigration, to the migration of all of the permanent canine teeth (1-20).

We analyzed a variable population; therefore, the parameters not mentioned were marked with the nomenclature "NM" (not mentioned).

With regard to the results obtained, as for the diagnosis 


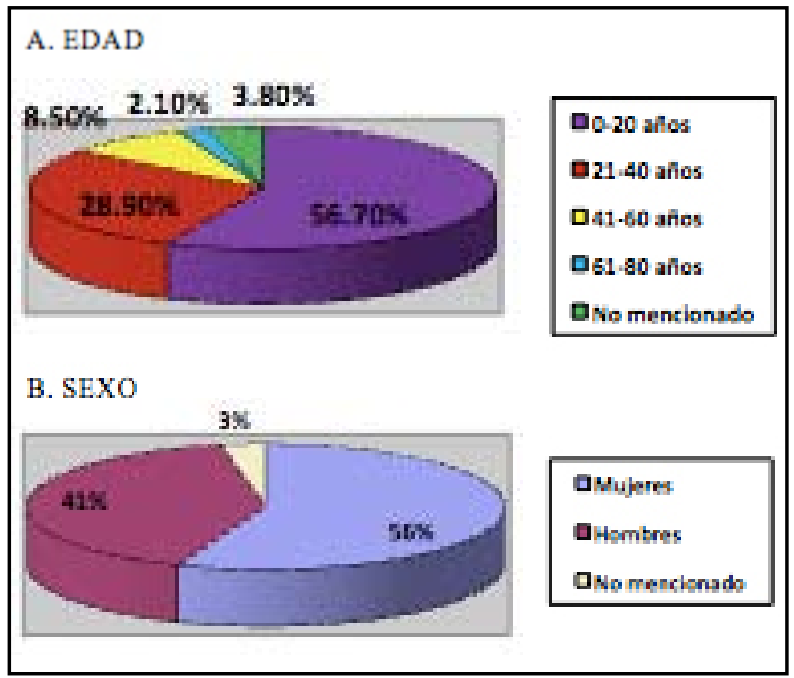

Fig. 1. A-Distribution of patients with presence of transmigrated canines in relationship to age.

$B$-Distribution of patients by sex.

of transmigrated canines, we observed that the patients ranged in age from 8 to 74 years old, with the mean age being $21.9 \pm 13.4$ years old. The greatest prevalence was found in the age group that ranges from 0 to 20 years old, which accounted for $56.7 \%$ of the sample. These canines are diagnosed in adolescence in the majority of cases, with the next age group between 20 and 40 years old, accounting for $28.9 \%$ of the sample, with this percentage decreasing as the age increases (Fig. 1a).

In terms of the patient's sex, we evaluated 187 patients with presence of transmigrated canines, 105 were women (56\%), 76 were men (41\%), and in 6 cases $(3 \%)$ the patient's sex was not mentioned (Fig. 1b).

As for the location of the canine that most often transmigrates, we observed that among the 209 transmigrated canines, $89.9 \%$ were mandibular canines (188 canines). It must be mentioned that $8 \%$ of the transmigrations were bilateral (both mandibular canines), and there was one case in which all of the canines had transmigrated.

The canine that most often transmigrates is number 33 in $52.7 \%$ of the patients ( 110 canines), followed by number 43 in $37.3 \%$ of the patients ( 78 canines). Tooth number 23 transmigrated in $2.9 \%$ of the patients ( 6 cases), and tooth number 13 transmigrated in $2.4 \%$ of the patients ( 5 cases). In $4.7 \%$ of the patients ( 10 cases), it is not mentioned which canine is the one that transmigrated (Fig. 2).

As for the presence of the deciduous canine, we observed that in 100 cases, the deciduous canine was present in the mouth (48\%); it had exfoliated in 69 cases (33\%), and its location was not mentioned in 40 cases (19\%). With regard to the position in which it was found, we distributed them according to the Mupparapu classification (21), who classifies them into five types:

- Type 1: Impacted canine that is mesially inclined

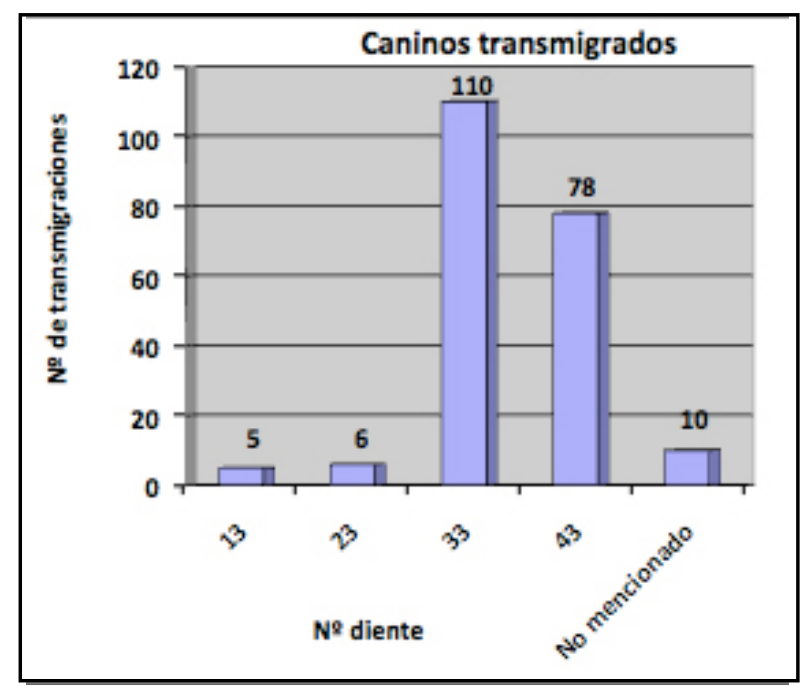

Fig. 2. Number of transmigrated canines.

through the midline, lip line or lingual line to the front teeth.

- Type 2: Horizontally impacted canine next to the mandibular ridge, below the apices of the incisors.

- Type 3: Canine that erupts on the contralateral side (the contralateral canine moving mesially or distally). - Type 4: Horizontal impacted canine next to the lower edge of the mandible, on the apices of the back teeth on the contralateral side.

- Type 5: Canine positioned vertically on the midline, with the longitudinal axis crossing the midline.

We found that in $46.9 \%$ of the cases, the transmigrated canine had passed the midline (type 1). In $25.4 \%$ of the cases, the transmigrated canine was in a horizontal position below the apices of the lower incisors (type 2). In $14.3 \%$ of the cases, the canine was located under the apices of the back teeth on the contralateral side (type 4). In $5.3 \%$ of the cases, the transmigrated tooth erupted on the contralateral side (type 3 ), and only in $1.4 \%$ of the cases was the transmigrated tooth found in a vertical position on the midline (type 5 ). In $0.5 \%$ of the cases, its extraoral eruption was described, and in $6.2 \%$ of the cases, it was not mentioned in what position it was found (Fig. 3).

Finally, we analyzed the pathologies associated with the presence of the transmigrated canine. Along the same lines, we found that $17.2 \%$ of the cases presented associated cystic pathology. This percentage includes those cases in which an intraoral fistula was found. A smaller percentage of other pathologies were found, such as the presence of odontomas in $3.3 \%$ of the cases, or the presence of supernumerary teeth in $1.9 \%$ of the cases. In $0.5 \%$ of the cases, there was a mandibular fracture, and the canine was resorbed in the same proportion. In $9 \%$ of the cases (19 patients), there is no reference to the presence or absence of pathology. Finally, in $67.5 \%$ of the cases (141 patients), we did not observe any pathology. 


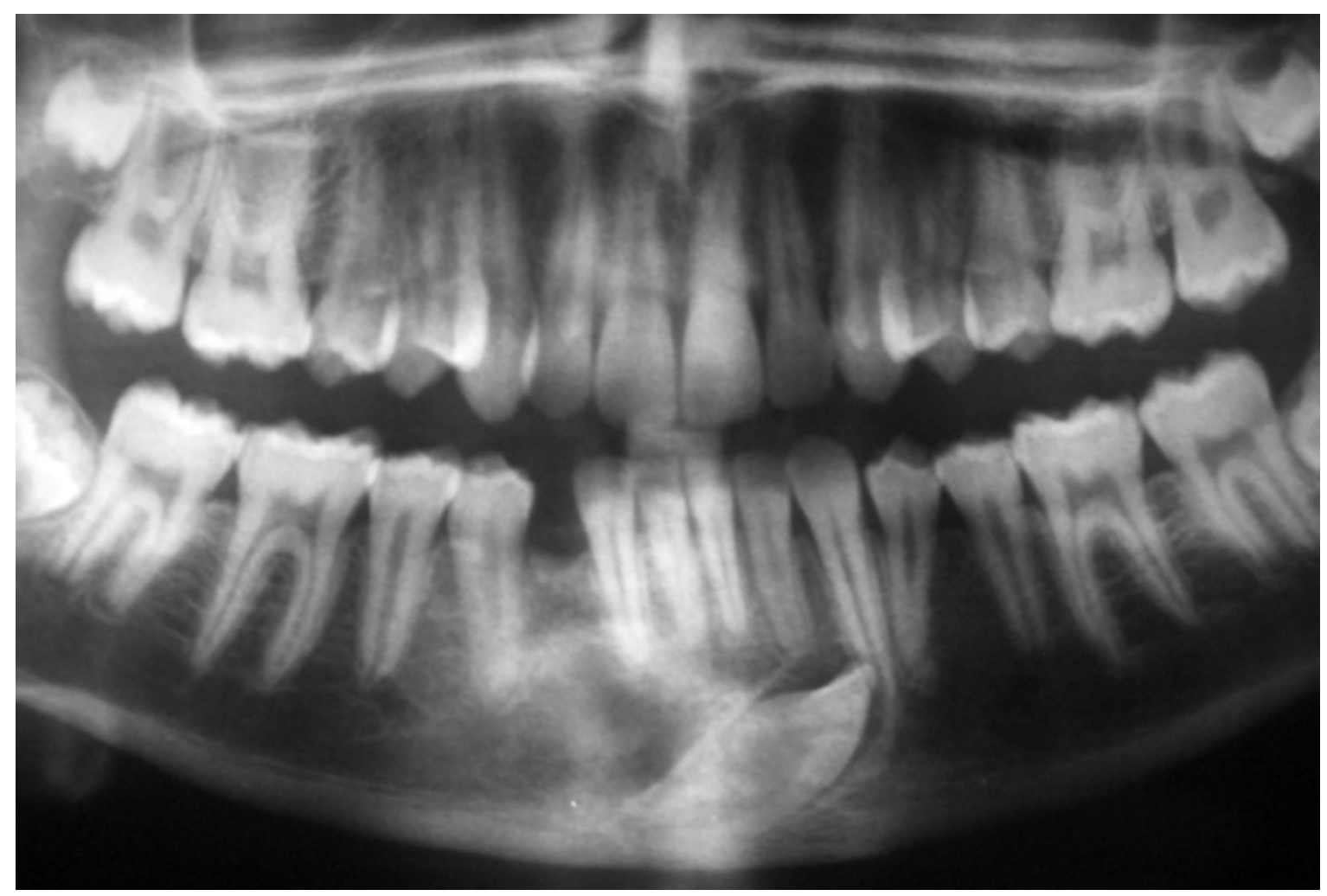

Fig. 3. Panoramic radiography showing an example of a transmigrated canine (43).

\section{Discussion}

It is important to keep in mind that when it comes to comparing their results, there are very few documented cases of the presence of transmigrated canines.

With respect to the age of the patient when finding these canines, they could be diagnosed at different ages. For Torres Lagares et al. (1), the age range is between 8 and 62 years old; whereas for González-Sánchez et al. (3), the range was up to 69 years old. However, all of the authors concur that they are most often diagnosed between the first and second decades of life. The eldest patient in our review, a 74-year-old patient, was presented by Aydin et al. (13).

The canines transmigrate during their physiological stage of eruption. The only thing that varies among different authors is the age when such migration is diagnosed. With respect to the patient's sex, there is great unanimity among the majority of the authors. All of the authors agree that there is a higher prevalence of this accident among women $(2,3,6-8,10-12)$. There is no plausible reason why it occurs more often in women. That is why authors such as Joshi et al. (8) and Buyukkurt et al. (7) believe that it is due to the fact that a greater number of women versus men visit the orthodontic clinic, which is where the diagnosis is most often made. Along this same line, our review shows more women than men, with their prevalence being $56 \%$. Joshi et al. (8) presented a predominantly female sample, whereas Buyukkurt et al. (7),
Aydin et al. (13) and González-Sánchez et al. (3) had a predominantly male sample. Although that is the case, we must remember that their sample does not exceed 15 patients, in comparison to the 28 patients in the sample analyzed by Joshi et al. (8). Other authors with fewer patients, such as Al-Waheidi et al. (10), whose number of canines ranged from 8 to 15 , presented a higher number of female patients.

It should also be mentioned that although we do not know the sex for $3 \%$ of the patients in our study, this percentage would not change the greater prevalence found among women.

As for the canine that transmigrates most often, all of the authors concur that the mandibular canines are most predominant. With regard to their presentation, they also agree that it is most often found unilaterally $(2,3)$, but although the large majority of authors believe that the left mandibular canine is the one that most often transmigrates $(3,7,8,11)$, some authors believe that it is the right mandibular canine that most often transmigrates (11). For Torres Lagares et al. (1), the occurrence of bilateral transmigrated mandibular canines was $12 \%$ in their studies. In our review, we observed that it is a generally unilateral phenomenon (having been found in 169 patients), predominantly affecting the left mandibular canine and occurring bilaterally in the remaining 17 patients, with one case of a patient whose four canine teeth had transmigrated. 
As for the presence or absence of the deciduous canine, González-Sánchez et al. (3) affirm that the canine is in the mouth in $40-70 \%$ of the cases, whereas Joshi et al. (8) affirm that it is in the mouth in $70.8 \%$ of their studies. In our review, the deciduous canine was found in the mouth in $48 \%$ of the patients. Among the authors with more cases, we observed that González-Sánchez et al. (3) and Joshi et al. (8) showed a higher number of deciduous canines in the mouth, whereas Buyukkurt et al. (7) and Aydin et al. (13) had more cases in which the deciduous canine had exfoliated (both authors affirming this to represent $64.2 \%$ of the cases). Given that in $19 \%$ of the cases of our review, it is not mentioned whether the deciduous canine was present in the arcade or if it had exfoliated, we cannot affirm that the deciduous canine is found with more prevalence in the mouth.

In relationship to the position, Torres Lagares et al. (1) and Buyukkurt et al. (7) concur that the most common position is type 1 of the Mupparapu classification (21) (the canine crosses the midline in a mesio-angular position). The results of González-Sánchez et al. (3) and Joshi et al. (8) differ, given that both found a greater number of cases in the position type 2 (the canine is in a horizontal position under the apices of the lower incisors). Our review found a higher percentage in position 1 of Mupparapu (21), concurring with Torres Lagares et al. (1) in that the next position with the highest number of cases is position type 2 .

With regard to the presence of associated pathologies, the authors concur that some associated pathologies can be found, such as cysts, odontomas, supernumerary teeth, etc. Al-Waheidi et al. (10) believe that cysts are the most commonly associated pathology, given that six out of eight of their cases presented a cyst.

The majority of the authors concur that the absence of pathology is the most common, the most common pathology present being that of radiolucency compatible with a cyst. For Joshi et al. (8), 35.7\% of transmigrated canines occur along with an associated cyst. Buyokkurt et al. (7) and Aydin et al. (13) also report cysts as being the first associated pathology. The only author who presents a higher number of another type of associated pathology is González-Sánchez et al. (3). In our review, we observed that $67.5 \%$ of the cases are not associated with any pathology, whereas $17.2 \%$ of the cases were associated with a cyst.

\section{Bibliography}

1. Torres Lagares D, Flores Ruíz R, Infante Cossío P, García Calderón M, Gutierrez Pérez JL. Transmigración del canino inferior incluido. Presentación de un caso y revisión de la literatura. Med Oral Patol Oral Cir Bucal. 2006; 11: 109-12.

2. Joshi MR, Shetye SB. Transmigration of mandibular canines: A review of the literature and report of two cases. Quintessence Int. 1994; 25: 291-4.

3. González-Sánchez MA, Berini-Aytes L, Gay-Escoda C. Transmigrant impacted mandibular canines: A retrospective study of 15 cases. J Am Dent Assoc. 2007; 138: 1450-5.

4. Tarsitano JJ, Wooten JW, Burditt JT. Transmigration of nonerupted mandibular canines: report of cases. J Am Dent Assoc. 1971; 82: 1395-7.

5. Alaejos-Algarra C, Berini-Aytes L, Gay-Escoda C. Transmigration of mandibular canines: Report of six cases and review of the literature. Quintessence Int. 1998; 29: 395-8.

6. Shapira Y, Kuftinec MM. Intrabony migration of impacted teeth. Angle Orthod. 2003; 73: 738-43.

7. Buyukkurt MC, Aras MH, Caglaroglu M, Gungormus M. Transmigrant mandibular canines. J Oral Maxillofac Surg. 2007; 65: 2025-9.

8. Joshi MR. Transmigrant mandibular canines: A record of 28 cases and a retrospective review of the literature. Angle Orthod. 2001; 71: 12-22.

9. Wertz RA. Treatment of transmigrated mandibular canines. Am J Orthod Dentofacial Orthop. 1994; 106: 419-27.

10. al-Waheidi EM. Transmigration of unerupted mandibular canines: a literature review and report of five cases. Quintessence Int. 1996; 27: 27-31.

11. Costello JP, Worth JC, Jones AG. Transmigration of permanent mandibular canines. Br Dent J. 1996; 181: 212-3.

12. Kharbanda OP, Choudhury AR. Extreme transmigration of mandibular cuspid: report of two cases. J Clin Pediatr Dent. 1994; 18 : 307-8.

13. Aydin U, Yilmaz HH, Yildirim D. Incidence of canine impaction and transmigration in a patient population. Dentomaxillofac Radiol. 2004; 33: 164-9.

14. Mitchell L. Displacement of a mandibular canine following fracture of the mandible. Br Dent J. 1993; 174: 417-8.

15. Kuftinec MM, Shapira Y, Nahlieli O. A case report. Bilateral transmigration of impacted mandibular canine. J Am Dent Assoc. 1995; 126: $1022-4$.

16. Shapira Y, Kuftinec MM. Intraosseous transmigration of mandibular canines- review of the literature and treatment options. Compend Contin Educ Dent. 1995; 16: 1014, 1018-20,1022-4; quiz 1026.

17. Milano M, Barrett L 2nd, Marshall E. Extraction of a horizontally impacted mandibular canine through a genioplasty approach: report of a case. J Oral Maxillofac Surg. 1996; 54: 1240-2.

18. Taguchi Y, Kurol J, Kobayashi H, Noda T. Eruption disturbances of mandibular permanent canines in Japanese children. Int J Paediat Dent. 2001; 11: 98-102.

19. Rebellato J, Schabel B. Treatment of a patient with an impacted transmigrant mandibular canine and a palatally impacted maxillary canine. Angle Orthod. 2003; 73: 328-36.

20. Shapira Y, Kuftinec M. Unusual intraosseous transmigration of a palatally impacted canine. Am J Orthod Dentofacial Orthop. 2005; 127: 360-3.

21. Mupparapu M. Patterns of intra-osseous transmigration and ectopic eruption of mandibular canines: review of literature and report of nine additional cases. Dentomaxillofac Radiol. 2002; 31: 355-60. 